Modeling response times in the size-congruity effect: Early vs. late interaction

Kristen A. Bowman and Thomas J. Faulkenberry

Tarleton State University

Author Note

Kristen A. Bowman and Thomas J. Faulkenberry, Department of Psychological Sciences, Tarleton State University.

All materials for replicating this study and the analysis are available for download. The raw data is available at https://git.io/JIU74, and all experiment and analysis scripts are available at https://osf.io/4mj5v/.

Correspondence concerning this article should be addressed to Kristen A. Bowman, Department of Psychological Sciences, Tarleton State University, Stephenville, TX 76402.

Email: kbowman@tarleton,edu 


\begin{abstract}
The size-congruity effect occurs when numerical magnitude interferes with judgments of physical size. Various accounts propose that this interference is either encoding-related or decision-related, though at present a clear consensus is lacking. In our study, we administered a single-digit physical comparison task (i.e., which digit is physically larger?) and applied four different mathematical models (ex-Gaussian, ex-Wald, shifted Wald and EZ-diffusion) to the observed response times. The aim of this modeling was to index the underlying cognitive processes via estimates of drift rate, response threshold, and non-decision time. The collection of estimates for each individual was then subjected to Bayesian paired samples $t$-tests. We found that the drift rate for incongruent trials was smaller than for congruent trials, indicating that congruent trials had a faster rate of information uptake. The response threshold for incongruent trials was generally larger than for congruent trials, indicating that for incongruent trials more information needed to be accumulated before a response could be initiated. Critically, we found evidence of an invariance in non-decision times between incongruent and congruent trials. This combination of results provides support for a late interaction account of the size-congruity effect, shedding further light onto models of decision making in number processing.

Keywords: Size-congruity effect, response time modeling, accumulator models, Wald distribution, EZ-diffusion model
\end{abstract}




\section{Modeling response times in the size-congruity effect: Early vs. late interaction}

The curious nature of decision making has intrigued cognitive psychologists over the past several decades. To better understand the complex processes involved in making decisions, experimenters usually limit the stimulus sets to the most basic units of information (e.g., words, letters, numbers, etc.). In this paper, our goal was to study the cognitive processes involved in decision making with numbers, and specifically the interference between physical and numerical magnitude known as the size-congruity effect (Henik \& Tzelgov, 1982).

The size-congruity effect is similar to the famous Stroop effect (Stroop, 1935; MacLeod, 1991), but with Arabic number digits rather than colors. Though the size-congruity effect may arise from several different experimental manipulations, we focus our attention on tasks where participants are asked to judge physical magnitude. In such a task, participants are presented with two single-digit number symbols where one symbol is physically larger than the other.

Participants are instructed to select the physically larger symbol but ignore numerical magnitude (Santens \& Verguts, 2011; Schwarz \& Heinze, 1998; Reike \& Schwarz, 2017). The task is comprised of two types of trials: congruent trials, where the physically larger symbol also has a larger numerical value (e.g. 3 vs 7 ) and incongruent trials, where the physically larger symbol has a smaller numerical value (e.g. 3 vs 7). For congruent trials, where physical size and magnitude lead to the same decision, participants are usually able to respond rather quickly. However, for incongruent trials, where physical size and magnitude lead to different decisions, participants typically require more time before a response is finalized. This size-congruity effect demonstrates that participants form a representation of numerical magnitude even when it is not needed to complete the task. Though the effect has been demonstrated numerous times (Algom, Dekel, \& Pansky, 1996; Besner \& Coltheart, 1979; Faulkenberry, Vick, \& Bowman, 2018; 
Fitousi \& Algom, 2006; Henik \& Tzelgov, 1982; Sobel, Puri, \& Faulkenberry, 2016; Sobel, Puri, Faulkenberry, \& Dague, 2017; Reike \& Schwarz, 2017; Risko, Maloney, \& Fugelsang, 2013), we still do not fully understand why it occurs.

\section{Early vs. Late Interaction}

Rather than questioning "if" the size-congruity effect occurs, we are more interested in "how" it occurs, and thus seek to uncover the mechanisms that are responsible for the interference between numerical and physical magnitude. To date, there has been conflicting evidence concerning the locus of this interference. In one of the first papers to explicitly model this interference, Santens and Verguts (2011) proposed that the size congruity effect stems from either an input level interference, reflecting representational overlap, or an output level interference, reflecting response competition. They framed these two accounts as an early interaction account and a late interaction account. An early interaction account suggests that the interference in incongruent trials occurs at the encoding stage. During this stage, participants encode both physical size and numerical magnitude into a shared analog representation (e.g., Walsh, 2003), which is then processed further and fed into a decision stage. On the other hand, a late interaction account suggests that the interference occurs not at encoding, but later at the decision stage. In this account there are two separate pathways for numerical input and physical input which both initiate separate numerical and physical representations. Both pathways then activate subresponses - when these two subresponses are in agreement (i.e., as in congruent trials), decision selection occurs rather quickly. However, when the two subresponses are in disagreement (i.e., as in incongruent trials), decision selection occurs more slowly (Santens \& Verguts, 2011; Schwarz \& Heinze, 1998). 
Equivocally, both the early interaction and late interaction accounts have received support in the literature. Schwarz and Heinze (1998) had participants perform a size congruity task and examined event-related potentials (ERPs) and lateralized readiness potentials (LRPs). They found reliable effects of congruity in frontal ERPs only 280 milliseconds after stimulus presentation. Further, they found no LRPs toward the incorrect response in incongruent trials, thus demonstrating an absence of a key signature of response competition. Both of these results led Schwarz and Heinze to propose that the locus of interference is in the early portion of the decision-making process.

On the other hand, Cohen Kadosh et al. (2007) examined the size-congruity effect with both ERPs and fMRI and found several signatures pointing to late interaction. First, they found interference-related activity in the primary motor cortex stemming from the hemisphere on the same side as the hand used for correct responses. This led Cohen Kadosh et al. (2007) to conclude that the congruity-related interference was not resolved until response initiation. Further, in contrast to Schwarz and Heinze (1998), Cohen Kadosh et al. (2007) found LRP deflection toward the incorrect response on trials when numerical distance was large (but not when distance was small). Both of these signature point to a late interaction account, though Cohen Kadosh et al. pointed out that late interaction only occurred when cognitive workload was low - when workload was high (i.e., small numerical distance), representational overlap would be sufficient to cause the size congruity effect.

Faulkenberry, Cruise, Lavro, and Shaki (2016) used computer mouse tracking to track the temporal dynamics of the size-congruity effect. Mouse trajectories on incongruent trials were significantly attracted toward the incorrect response throughout a large portion of the normalized 
response duration. This pattern of continuous, graded trajectory deflection was taken as evidence of response competition between the pathways of physical and numerical representation $-\mathrm{a}$ signature of the late interaction account.

\section{Response time models}

At this point, the mechanisms underlying the size-congruity effect remain unclear. One promising approach to disentangle these competing accounts is to use response time modeling to decompose distributions of response times into components which may shed light onto the relevant cognitive processes. Classically, response time modeling in cognitive psychology has used the ex-Gaussian distribution (Luce, 1986; Rieger \& Miller, 2019; Whelan, 2008). The term “ex-Gaussian" refers to an exponentially modified Gaussian distribution - that is, it contains a normal (Gaussian) component convolved with an exponential tail. This results in three components that can be extracted from the response time distribution: $\mu$, the mean of the normal component; $\sigma$, the standard deviation of the normal component; and $\tau$, the rate of the exponential tail. To see the utility of this approach, consider Penner-Wilger, Leth-Steensen, and LeFevre (2002), who used ex-Gaussian modeling to compare mental arithmetic performance across cultures. Specifically, they compared the problem-size effect (i.e., larger problems, such as 7 x 8 or $9+7$, require more time to answer than smaller problems, such as $3 \times 3$ or $3+2$ ) between Chinese and Canadian students. Penner-Wilger et al. found that for the Chinese group, the problem size effect occurred in the parameter $\mu$, whereas for the Canadian group, the effect occurred in both $\mu$ and $\tau$. Penner-Wilger et al. interpreted this pattern as follows: for the Chinese group, the locus of the problem size effect is solely in the normal component of the response time distribution, and thus reflects processes related to memory retrieval. On the other hand, the 
Canadian group exhibited the effect in the normal component and the tail, reflecting both memory retrieval and nonretrieval processing.

As one can infer from the previous example, it is tempting to assume that these parameters directly reflect underlying cognitive processes. The history of such thinking goes well back - for example, Hohle (1965) claimed that $\mu$ reflects the motor-related portion of the decision process and that $\tau$ reflects the cognitive processes. Yet, there has been very little agreement on the exact process that each parameter reflects, if any. In fact, it is now widely agreed that the ex-Gaussian distribution parameters should be viewed as solely a descriptive tool and should not be used to index cognitive processing (Matzke \& Wagenmakers, 2009; Schwarz, 2001). If we are to use response time modeling to uncover the cognitive processes underlying the size-congruity effect, we will need different tools.

\section{Wald distributions}

Our primary goal is to convert our observed response times into information about the unobservable, latent processes involved in decision making. One tool that has potential to meet this goal is an accumulator model (Link, 1975), which is a mathematical model that represent the decision-making process as a random walk towards a fixed response boundary (see, for example, Figure 1). These models represent decision making as the process of gathering noisy partial information over time until enough information is accumulated to make a decision. Typically, accumulator models use three basic parameters to index cognitive processes: quality of presented information, amount of information uptake required to trigger a response, and nondecision time (Anders, Alario, \& Maanen, 2016).

One such accumulator model is the Wald model (also known as the inverse Gaussian distribution). The Wald model is a distribution of stopping times for a continuous diffusion 
process (i.e., a continuous random walk) with a fixed boundary. The Wald distribution has two parameters: a drift rate $\gamma$ and a response threshold $\alpha$. The drift rate represents the rate at which the accumulator constantly tends toward the response boundary. In application, it is used to index the quality of presented information - stimuli with "higher quality" yield larger drift rates. The response boundary represents the value at which the accumulator stops. In application, it is used to index the amount of stimulus information required in order to trigger a decision - stimuli that require more information yield larger response boundaries.

While easy to specify mathematically, the Wald distribution has one problem - because its density function begins at zero, it places a positive likelihood on very small response times (i.e., less than 100 milliseconds). This is problematic because response times below 100 milliseconds are not reasonable to expect in a typical decision-making context (Luce, 1986). To correct for this, Schwarz (2001) recommended additively decomposing response times as $R T$ $=D+M$, where $D$ represents a decision component, and $M$ represents a nondecision component (i.e., the sum of all processes leading up to and following the decision process). In this context, we can use the Wald distribution to model $D$, but what do we use to model the nondecision component $M$ ? There are two possibilities: (1) we can construct an exponential modification of the Wald distribution; or (2) add a constant shift to the distribution.

The first of these results in the ex-Wald model (Schwarz, 2001). The ex-Wald model is a convolution of the Wald distribution with an exponential tail. This decomposes the response time distribution into two components: a decision component $D$ (modeled by the Wald distribution) and a nondecision component $M$ (modeled by an exponential distribution). The decision component $D$ has the two parameters described earlier: drift rate $\gamma$ and response threshold $\alpha$. The nondecision component $M$ has one parameter: the exponential rate parameter $\tau$. 
The second of these results in the shifted Wald model (Anders et al., 2016). The shifted Wald model moves mass away from small response times by simply adding a constant horizontal shift to the Wald density function - this constant shift is then assumed to represent the time accounting for the nondecision component. This results in a three-parameter decomposition of response time distributions: a drift rate $\gamma$, a response threshold $\alpha$, and a shift parameter $\theta$.

Whereas the ex-Wald model has received relatively little attention in the literature, there have been several applications of the shifted Wald model. We quickly mention two of these which have been used in the context of numerical cognition. Faulkenberry (2017) used the shifted Wald model to investigate the independence of encoding and calculation processes in mental arithmetic. He found that drift rate was affected by both problem size and problem format, indicating that the encoding variables (i.e., problem format) can penetrate downstream and directly affect calculation processes (e.g., Campbell \& Fugelsang, 2001; Frampton \& Faulkenberry, 2019).

Recently, Faulkenberry, Vick, and Bowman (2018) fit response times from a size congruity task to the shifted Wald model. They found a congruity effect on the decision related parameters: drift rate $\gamma$ and response threshold $\alpha$. Specifically, congruent trials had a higher drift rate $\gamma$ than incongruent trials, which indicated that congruent stimuli had a higher quality of information than incongruent stimuli. Also, congruent trials had a lower response threshold $\alpha$ than incongruent trials, which indicated that congruent trials induced a more liberal level of response caution than incongruent trials. Critically, there was no difference in the nondecision time $\theta$, indicating that the locus of the size-congruity effect is in the decision stage of processing, not in the encoding stage. 


\section{EZ-Diffusion Model}

The ex-Wald and shifted Wald are single boundary accumulator models - that is, they are designed to model noisy accumulation toward a single response boundary. Because there is only one threshold, we can only use the models with a single response (i.e., modeling correct responses only). Thus, we must exclude error trials from analysis. This is problematic because errors have the potential to hold rich information about the decision-making process. In order to include error trials in our analysis, we need an accumulator model with two boundaries - one to represent the noisy drift toward a correct response, and the other to represent the accumulation process toward an incorrect response. Fortunately, the diffusion model (Ratcliff, 1978) is perfectly suited for such an application. The full diffusion model has been the subject of much investigation over the past 40 years. Most instantiations of the diffusion model contain 7 parameters which are well known reflect various cognitive processes (Matzke, Wagenmakers, 2007; Ratcliff, Thapar, \& McKoon, 2001; Ratcliff \& Rouder, 2000; Wagenmakers, Ratcliff, Gomez, \& McKoon, 2008; Voss, Rothermund, \& Voss, 2004).

While Ratcliff's (1978) diffusion model provides a wealth of information about response time distributions, fitting the model can be quite difficult. Wagenmakers, Van Der Maas, \& Grasman (2007) used some simplifying assumptions to reduce the full seven-parameter diffusion model to a three-parameter EZ-diffusion model that can be estimated directly from three summary statistics: the mean and variance of the distribution of response times for correct decisions, and the overall proportion of correct responses. As with the Wald models above, the three parameters of the EZ-diffusion model are drift rate, response threshold, and nondecision time. 


\section{The Present Study}

The purpose of the present study was to use response time models to examine the locus of interference in size-congruity effect. We fit the observed response times from a physical comparison task to each of the four response time models described above: (1) the ex-Gaussian; (2) the ex-Wald; (3) the shifted Wald; and (4) the EZ-diffusion. We used these parameter estimates as the latent behavioral signatures of congruity effects on decision and nondecision processes. This allowed us to shed some light on the nature of the size-congruity effect specifically, whether the size-congruity effect is best explained by an early interaction account or a late interaction account.

Based on the models we described above, we were able to make the following a priori predictions from each account:

1. ex-Gaussian model: Since the ex-Gaussian is not a process model, the parameters do not map directly to cognitive processes (Matzke \& Wagenmakers, 2009). Nonetheless, Matkze and Wagenmakers (2009) observed specific correlations between the parameters of the ex-Gaussian and the EZ-diffusion parameters that may permit us to make tentative predictions. Specifically, they found that drift rate correlated negatively with all three exGaussian parameters $(\mu, \sigma, \tau)$, response threshold correlated positively with all three parameters, and nondecision time correlated positively with only $\mu$. Thus, the parameter $\mu$ is really the only parameter that can reasonably be expected to follow any specific pattern reflecting evidence for either the early or late interaction account. Specifically, if the size-congruity effect stems from early interaction, we expect congruity effects to persist in nondecision time; thus, we would expect $\mu$ to be larger for incongruent trials compared to congruent trials. On the other hand, a late interaction account would keep 
congruity effects localized in the decision components, which may have effects on all three parameters. Thus, the reader is cautioned to note that any observed congruity effects on $\mu, \sigma$, or $\tau$ do not provide any strong evidence for either account. As such, we do not use the ex-Gaussian model in any confirmatory sense. But, for completeness, we present the results of our ex-Gaussian modeling as purely exploratory results.

2. ex-Wald model: the critical parameter is $\tau$, the mean of the exponential nondecision component. If the size-congruity effect stems from early interaction - that is, shared representational overlap before feeding into a decision stage - we would expect $\tau$ to be larger for incongruent trials than for congruent trials, and the decision-related parameters (drift rate and response threshold) to remain invariant to the congruity manipulation. On the other hand, if the size-congruity effect stems from late interaction - that is, response competition during the decision process - we would expect the opposite pattern. Namely, for incongruent trials we should observe a decrease in drift rate and/or an increase in response threshold, and the nondecision parameter $\tau$ should remain invariant.

3. shifted-Wald model: the predictions are similar to the predictions of the ex-Wald model above. Specifically, the early interaction account would be supported if we observe a congruity related increase in nondecision time and an invariance for drift rate and response threshold. On the other hand, the late interaction account would be supported if we instead observe a congruity related decrease in drift rate and/or an increase in response threshold along with (critically) an invariance in nondecision time.

4. EZ-diffusion model: the predictions are identical to the predictions of the shifted Wald. However, we include the EZ-diffusion in our analysis for two reasons. First, even though participants typically commit very view errors in this task, we believe the ability to 
include errors in our analysis is much more principled than simply ignoring them.

Second, as Matzke and Wagenmakers (2009) cast some doubt on the ability of the shifted Wald model to provide an interpretable index of cognitive processing (but see Anders et al., 2016), including the EZ-diffusion estimates of drift rate, response threshold, and nondecision time along with our shifted-Wald estimates will provide an additional test to support our Wald-related theorizing about the early/late nature of the size-congruity effect.

\section{Method}

\section{Participants}

A total of 53 undergraduate students (20 males, 33 females, mean age 19.45 years, $\mathrm{SD}=$ 1.44 years) were recruited to participate in this experiment and were offered partial course credit.

\section{Stimuli and Procedure}

Participants were presented with pairs of single-digit Arabic numerals chosen from the stimulus set $(2,3,4,5,6,7$, and 8$)$. In order to balance numerical distance between numerals, the following 12 pairs were selected: 2-3, 3-4, 4-5 (distance 1); 2-4, 3-5, 4-6 (distance 2); 2-5, 3-6, 47 (distance 3); 2-6, 3-7, 4-8 (distance 4). The primary manipulation was the font size of the stimuli. The physically larger digits were presented in 36-point font, whereas the physically smaller digits were presented in 28-point font (see Figure 2).

Thus, there were two different conditions, congruent trials and incongruent trials. For congruent trials, the physical size and magnitude led to the same decision (e.g., large font and large magnitude). For incongruent trials, the physical size and magnitude led to different decisions (e.g., large font and small magnitude). 
Each pair was presented in different left-right orders and different font configurations (smaller/left; larger/right; or smaller/right; larger/left). In total, there were 12 pairs $\mathrm{x} 2$ congruity conditions $\times 2$ orders $\mathrm{x} 2$ font configurations $=96$ experimental trials per block. Participants completed 4 blocks for a total of 384 trials per participant. The completion time for each participant was approximately 10 minutes.

Data was collected via the OpenSesame software package (Mathôt, Schreij, \& Theeuwes, 2012) and ran on a Lenovo Thinkpad X220 computer with a 12.5-inch display at a resolution of 1366 x 768 pixels. A standard Dell computer keyboard was used for responses. The "A" key represented the selection of the leftmost number on the computer screen, whereas the "L" key represented the selection of the rightmost number on the computer screen. Prior to the start of the experiment, students were instructed to select the physically larger of the two numbers as quickly and as accurately as possible.

Each trial started with a fixation point that was displayed for 500 milliseconds. Immediately after, the stimulus pair was presented. Each pair remained on the screen until a response was given. If participants selected the correct response (the physically larger number), they were given no feedback and the next trial began. However, if participants selected the incorrect response, they were given a red " $\mathrm{X}$ " for one second and the next trial began immediately after (see Figure 3).

Upon completion of the experimental trials, participants were given a short demographic survey and thanked for their participation. All anonymous raw data were uploaded nightly to GitHub under a "born-open” data archiving protocol (Rouder, 2015) and are available for download at https://git.io/JIU74. 


\section{General data workflow}

We applied the following basic workflow to each of our four response time models: (1) separation of trials into design cells; (2) parameter estimation; and (3) Bayesian model comparison. We now describe each of these steps in detail:

1. Separation into design cells. We separated the observed response times into 106 design cells, each resulting from combining the factors of participant $(N=53)$ and condition (congruent, incongruent).

2. Parameter estimation. We fit each of the four response time models (ex-Gaussian, ex-Wald, shifted-Wald, EZ-diffusion) to the distribution of response times contained in each of the design cells from Step 1. For the ex-Gaussian, ex-Wald, and shifted-Wald models, the fitting was done via maximum likelihood estimation (Myung, 2003; Heathcote, 2004). For the EZ-diffusion model, we used the closed-form technique of Matzke and Wagenmakers (2007). Each model fit yielded 3 parameters to describe the distribution of congruent trials and an additional 3 parameters for the distribution of incongruent trials. For each model, this gave us a collection of 6 parameter estimates from each participant (318 parameter estimates total).

3. Bayesian model comparison. To assess the effect of our congruity manipulation on the population-level parameters in our response time models, we used Bayesian paired samples $t$-tests (Rouder, Speckman, Sun, Morey, \& Iverson, 2009). Among the many practical advantages of Bayesian testing over traditional frequentist testing (Wagenmakers, 2007; Faulkenberry, Ly, \& Wagenmakers, 2020), the Bayesian $t$-test provides us with one major advantage. Since our theory 
testing involves specifically testing for patterns of invariance among the observed parameter estimates, we need to be able to provide evidence for specific null hypotheses (something frequentist testing cannot do). We measure evidence for specific hypotheses using Bayes factors (Kass \& Raftery, 1995), which measure the predictive adequacy of two competing hypotheses. A Bayes factor indexes the extent to which the observed data are more likely under one hypothesis compared to another. For example, a Bayes factor of $\mathrm{BF}_{01}=10$ for the null hypothesis would indicate that the observed data are 10 times more likely under the null hypothesis than an alternative hypothesis. If we assume 1-to-1 prior odds for the null and alternative, we can convert an obtained Bayes factor into a posterior probability for the null by computing $p\left(\mathcal{H}_{0} \mid\right.$ data $)=\frac{B F_{01}}{1+B F_{01}}=\frac{10}{1+10}=0.91$ (see Masson, 2011).

For our Bayesian $t$-tests, we defined competing hypotheses about the population-level effect size $\delta$ of our congruity manipulation. For the accumulator models, we have specific predictions about the direction of congruity-related effects (if any) on the population-level parameters. For drift rate, our expected congruity effects are negative (i.e., drift rate will decrease for incongruent trials). Thus, the model comparison is between $\mathcal{H}_{0}: \delta=0$ and $\mathcal{H}_{1}: \delta<0$. For response threshold and nondecision time, any congruity-related effects are expected to be positive. Thus, for these parameters the model comparison is between $\mathcal{H}_{0}: \delta=0$ and $\mathcal{H}_{1}: \delta>0$. Note that for the ex-Gaussian model, we do not employ any specific confirmatory prediction; as such, we use a two-sided alternative and compare $\mathcal{H}_{0}: \delta=0$ to $\mathcal{H}_{1}: \delta \neq 0$. All model comparisons were performed in 
JASP (JASP Team, 2020), and the associated JASP worksheet is available for download at https://osf.io/4mj5v/. Bayesian hypothesis testing further requires that we specify a prior distribution for $\delta$ under $\mathcal{H}_{1}$. We use the default Cauchy distribution with scale $r=\frac{1}{\sqrt{2}}$ (Rouder et al, 2009). This prior is well-calibrated, relatively noninformative, and forms a good "objective" prior to use across the wide variety of hypothesis tests that we performed ${ }^{1}$. For tests which are evidential for $\mathcal{H}_{1}$ (i.e., $\delta$ is nonzero), we computed a central $95 \%$ credible interval for $\delta$.

\section{Results}

Participants completed a total of 20,352 trials. We discarded 636 trials that contained an incorrect response (error rate $=3.125 \%$ ). Further, we removed an additional 11 trials that were slower than 100 milliseconds and 71 trials that were longer than 2,000 milliseconds. This cleaning procedure resulted in retaining a total of 19,634 trials ( $96.47 \%$ of original trials) for further analysis ${ }^{2}$.

\section{Mean Response Times}

First, we analyzed the effect of the congruity manipulation on mean response times. As expected, we found compelling evidence for the size congruity effect on mean response times in the physical comparison task (see Figure 4). The mean of the response time distribution for incongruent trials $(M=620.1, S D=114.6)$ was greatly increased compared to the distribution for congruent $(M=554.3, S D=104.4)$ trials, indicating that incongruent trials required more processing time than congruent trials. This is confirmed by a Bayes factor of $\mathrm{BF}_{10}=2.078 \mathrm{x}$

\footnotetext{
${ }^{1}$ For each model comparison, we performed a sensitivity analysis using a range of reasonable values for $r$. For clarity, we only report the Bayes factor associated with $r=1 / \sqrt{2}$, but the general conclusions remained the same for all other scale values.

${ }^{2}$ For the EZ-diffusion model, we retained the error trials to compute the proportion of correct trials.
} 
$10^{17}, p\left(\mathcal{H}_{1} \mid\right.$ data $)>0.999,95 \% \mathrm{CrI}=[1.50,2.44]$. In all, we see a substantial congruityrelated increase in mean response times.

Next, we attempted to more fully describe the effects of physical-numerical congruity on the distributions of response times. To this end, we fit the distributions of response times for each participant with four different response time models and then tested specific hypotheses about the population-level effect size on each parameter under our congruity manipulation. We now present the results of this modeling. The mean and standard deviation of each parameter estimate can be seen in Table 1.

\section{ex-Gaussian modeling}

As we described earlier, the results of our ex-Gaussian modeling are purely exploratory, and we include them here for completeness. As such, we make no claims about cognitive processing based on these results. The mean of the ex-Gaussian parameter $\mu$ was larger for incongruent trials $(M=443.6)$ than for congruent trials $(M=425.3), \mathrm{BF}_{10}=910.9, p\left(\mathcal{H}_{1}\right.$ । data $)=0.999,95 \% \mathrm{CrI}=[0.32,0.91]$. The mean of the ex-Gaussian parameter $\sigma$ was larger for incongruent trials $(M=57.09)$ than for congruent trials $(M=46.24), \mathrm{BF}_{10}=307.3, p\left(\mathcal{H}_{1}\right.$ । data $)=0.997,95 \% \mathrm{CrI}=[0.28,0.86]$. Finally, the mean of the ex-Gaussian parameter $\tau$ was larger for incongruent trials $(M=176.6)$ than for congruent trials $(M=128.9), \mathrm{BF}_{10}=1.21 \mathrm{x}$ $10^{15}, p\left(\mathcal{H}_{1} \mid\right.$ data $)>0.999,95 \% \mathrm{CrI}=[1.32,2.20]$

\section{ex-Wald modeling}

Recall from our earlier discussion that we were able to make specific directional hypotheses for each parameter of the accumulator models. For convenience, we outline them here again. First, we predict that the congruity effect (if any) on drift rate will be negative - that is, we define $\mathcal{H}_{1}: \delta<0$. For response threshold, we expect congruity effects (if any) to be 
positive - that is, we define $\mathcal{H}_{1}: \delta>0$. Finally, for nondecision time, we expect congruity effects (if any) to be positive; that is, we define $\mathcal{H}_{1}: \delta>0$.

First, we considered the ex-Wald drift rate. We found that the mean drift rate was larger for congruent trials $(M=0.4495)$ than for incongruent trials $(\mathrm{M}=0.3808), \mathrm{BF}_{10}=147.5, p\left(\mathcal{H}_{1} \mathrm{I}\right.$ data $)=0.993,95 \% \mathrm{CrI}=[-0.79,-0.22]$. This confirms our prediction that the rate of information uptake decreased for incongruent trials. The mean response threshold for congruent trials $(M=187.9)$ was not smaller than the mean response threshold for incongruent trials $(M=$ 164.5), $\mathrm{BF}_{01}=27.80, p\left(\mathcal{H}_{0} \mid\right.$ data $)=0.966$. This indicated reasonably strong evidence for a null effect of congruity on response threshold, as the observed data were approximately 29 times more likely under the null hypothesis than the alternative hypothesis. Finally, for the nondecision parameter of the ex-Wald, the mean for congruent trials $(M=123.4)$ was smaller than the mean for incongruent trials $(M=163.8), \mathrm{BF}_{10}=2.47 \times 10^{6}, p\left(\mathcal{H}_{1} \mid\right.$ data $)>0.999,95 \% \mathrm{CrI}=[0.58$, 1.23]. This indicated that for the nondecision parameter there is a positive congruity effect. Thus, our pattern of results with the ex-Wald model are equivocal - whereas we found substantial evidence of a congruity effect on the nondecision parameter, we also found a congruity effect on drift rate. These results do not point clearly to either the early or late interaction account, so let us consider the final two accumulator models.

\section{shifted Wald modeling}

The mean shifted-Wald drift rate was larger for congruent trials $(M=0.13)$ than for incongruent trials $(M=0.11), \mathrm{BF}_{10}=1.97 \times 10^{6}, p\left(\mathcal{H}_{1} \mid\right.$ data $)>0.999,95 \% \mathrm{CrI}=[-1.22,-$ 0.57]. This indicates that the rate of information accumulation from congruent trials was faster compared to incongruent trials. The mean response threshold was only very slightly smaller for congruent trials $(M=39.68)$ than for incongruent trials $(M=41.28)$; this observed difference was 
more likely under a null hypothesis, $\mathrm{BF}_{01}=3.657, p\left(\mathcal{H}_{0} \mid\right.$ data $)=0.785$. Critically, the mean nondecision time for congruent trials $(M=252.1)$ did not increase for incongruent trials $(M=$ 238), $\mathrm{BF}_{01}=14.73, p\left(\mathcal{H}_{0} \mid\right.$ data $)=0.936$. This indicates that the observed nondecision times were approximately 15 times more likely under the null hypothesis than the alternative hypothesis. This gives us positive evidence for the absence of a congruity effect on nondecision time. With this null effect and the observed positive congruity effect on drift rates, our shifted Wald modeling supports a late interaction account of the size congruity effect.

\section{EZ-diffusion modeling}

Similar to what we observed with the shifted Wald model, the mean EZ-diffusion drift rate was larger for congruent trials $(M=0.01102)$ than for incongruent trials $(M=0.009511)$, $\mathrm{BF}_{10}=1.31 \times 10^{11}, p\left(\mathcal{H}_{1} \mid\right.$ data $)>0.999,95 \% \mathrm{CrI}=[-1.71,-0.76]$. This indicated that the rate of information accumulation for congruent trials was faster than for incongruent trials. Also, the mean response threshold was smaller for congruent trials $(M=5.489)$ than for incongruent trials $(M=6.283), \mathrm{BF}_{10}=3.21 \times 10^{12}, p\left(\mathcal{H}_{1} \mid\right.$ data $)>0.999,95 \% \mathrm{CrI}=[1.07,1.87]$. This indicated that participants required more information before making a decision on incongruent trials. Critically, the mean nondecision time for congruent trials $(M=296.2)$ did not increase for incongruent trials $(M=282), \mathrm{BF}_{01}=20.41, p\left(\mathcal{H}_{0} \mid\right.$ data $)=0.953$. These results mirror those from the shifted Wald model - namely, congruity had a negative effect on drift rate, a positive effect on response threshold, and a null effect on nondecision time. Again, these data support a late interaction account of the size congruity effect.

\section{Discussion}

The purpose of the present study was to use response time modeling to compare two theoretical models of the size-congruity effect. This classic phenomenon in numerical cognition 
(Besner \& Coltheart, 1979; Henik \& Tzelgov, 1982) occurs when participants exhibit an interference effect when asked to choose the physically larger of two numbers whose physical and numerical size are incongruent. Because the task could be completed by completely ignoring the semantic information of numerical magnitude and simply focusing on the visual template of the stimuli, the presence of the size-congruity effect is strong evidence that people simply cannot ignore this numerical information. Our main aim in this study was to investigate the locus of this interference effect. We follow Santens and Verguts (2011) and consider two accounts of the interference: an early interaction account, where the interference occurs at early stages of processing, and a late interaction accounts, where the interference reflects dynamic competition between parallel and partially active responses. We used response modeling to disentangle these two competing accounts of the size-congruity effect.

Our results demonstrated the expected size congruity effect. More importantly, we modeled the observed response times with four models: an ex-Gaussian model (Hohle, 1965; Luce, 1986), an ex-Wald model (Schwarz, 2001), a shifted-Wald model (Anders et al., 2016; Faulkenberry, 2017), and the EZ-diffusion model (Wagenmakers et al., 2007). With the exception of the ex-Gaussian model, these response time models allowed us to decompose observed response times into decision-related and nondecision components. With this decomposition, we were able to propose specific hypotheses about the effects of our congruity manipulation on each component. Specifically, an early interaction account would lead to congruity effects on nondecision parameters, but an invariance on decision-related parameters (e.g., drift rate and/or response threshold). On the other hand, a late interaction account would predict the opposite - namely, congruity effects on drift rate and/or response threshold, but critically, an invariance on nondecision parameters. To assess evidence for these effects and 
invariances, we used Bayesian hypothesis testing (Rouder et al., 2009; Wagenmakers, 2007; Faulkenberry et al., 2020).

For the ex-Gaussian distribution, we found congruity effects on all three parameters. We do not attempt to interpret these results as indicative of early versus late interaction, however, as the ex-Gaussian is purely a descriptive model rather than a process model. As such, we can make no specific claims about cognitive processes from the ex-Gaussian parameters. For the ex-Wald, the shifted Wald, and the EZ-diffusion models, we found a consistent congruity effect on drift rate, where congruent trials exhibited a faster rate of information uptake than incongruent trials. Whereas there is a consistent pattern for drift rate, we did not see consistency across congruity effects on response threshold. For the shifted Wald, we found that response threshold was invariant to our congruity manipulation. However, for both the ex-Wald and the EZ-diffusion models, we found the expected positive congruity effect, where incongruent trials required more accumulated information before response than congruent trials. In spite of this inconsistency, these results tentatively indicate that the interference in the size-congruity effect occurs in the decision stage, possibly supporting a late interaction model over an early interaction model. The critical test for the late interaction account is whether the nondecision parameters are invariant to the congruity manipulation.

It turns out that the answer is mostly "yes.” For the shifted Wald and EZ-Diffusion models we found positive evidence for an invariance of the congruity manipulation on nondecision time. For both of these models, our data were much more likely under a hypothesis of no congruity effect than they were under a hypothesis where incongruent trials increase the time required for nondecision-related processing (as would be expected in an early interaction account; Faulkenberry et al, 2018). For the ex-Wald model, we found an unexpected congruity 
effect on the nondecision component, but this was accompanied by a congruity effect on drift rate. We think the observation of congruity effects in both decision and nondecision components undermines any attempt to interpret the ex-Wald modeling as support for either account.

In all, we think our observed data points to the following: the locus of the size-congruity effect seems to be present in the decision-related stages of processing and not in the encodingstages. This conclusion is in line with several other studies (Faulkenberry et al., 2016; 2018; Sobel et al., 2016, 2017). These data provide further evidence for a late interaction account of the size-congruity effect, as originally proposed by Santens and Verguts (2011). Furthermore, the present study is novel in its use of systematic mathematical modeling to more fully describe response time distributions. Not only do our methods allow a more fine-grained analysis of the latent cognitive processes involved in a numerical task, they also yield a more principled approach to describing observed data. It is a common practice in response time studies to simply use participants' mean response times to make inferences about cognitive processes. However, by collapsing a distribution of response times to just one number, there is much information lost. We think using process models to describe response time distributions is a much better approach to investigating cognitive tasks.

One of the main limitations of these results is the apparent inconsistency in the observed congruity effects on response threshold - particularly the surprising null effect in the shifted Wald model. Based on our previous work with the size-congruity effect (Faulkenberry et al., 2018), we predicted that the response threshold for incongruent trials would be larger than the response threshold for congruent. While the invariance we observed was not expected, the evidence for this null effect was relatively small. This unexpected finding could be a good target for further investigation. Additionally, there is a debate concerning the interpretability of these 
parameters as indices of latent cognitive processes. Though there is clear justification for being caution about such interpretation (Matzke \& Wagenmakers, 2009), others have proposed that the parameters can indeed be interpreted as reflecting specific processing stages (Schwarz, 2001; Anders, Alario \& Van Maanen, 2016). Because of this inconsistency in the literature, we backed up our interpretations with the EZ-diffusion model (Matzke \& Wagenmakers, 2009), a simplification of the diffusion model, which is highly credible as a model of underlying cognitive processes (Ratcliff, Thapar, \& McKoon, 2001; Ratcliff \& Rouder, 2000;

Wagenmakers, Ratcliff, Gomez, \& McKoon, 2008; Voss, Rothermund, \& Voss, 2004). We think the similarities between our inferences from our Wald model approaches and the EZ-diffusion model provides further validation our claim that the size-congruity effect stems from late interaction.

Clearly, the results of this study need to be further replicated. One future extension of this study would be to investigate the methods used to fit the response time models. For example, we used basic maximum likelihood estimation (Myung, 2003; Heathcote, 2004) to fit the exGaussian, ex-Wald, and shifted-Wald models. Anders et al. (2016) used a fundamentally different method to fit the shifted Wald distributions in their work, and these functions were used in our previous work with shifted Wald models (e.g., Faulkenberry, 2017; Faulkenberry et al., 2018). Perhaps this is why we did not observe the expected congruity effect on response threshold. Ideally, these two fitting methods should lead to the same general outcome, but this would need to be further investigated. Further, in future work we wish to employ hierarchical Bayesian modeling (Farrell \& Ludwig, 2008) in order to avoid the need for collapsing parameter estimates across participants. 
From a wider scope, the size-congruity effect is reflective of cognitive processing in general. The size-congruity effect is just one of many experimental tasks that induce interference effects which help researchers theorize about general cognitive processes. By learning how these interferences occurs, psychologists can gain greater understanding of how people make decisions, not only with numbers, but with more complex information as well. 


\section{References}

Algom, D., Dekel, A., \& Pansky, A. (1996). The perception of number from the separability of the stimulus: The Stroop effect revisited. Memory \& Cognition, 24(5), 557-572. https://doi.org/10.3758/bf03201083

Anders, R., Alario, F.-X., \& Van Maanen, L. (2016). The shifted Wald distribution for response time data analysis. Psychological Methods, 21(3), 309-327. https://doi.org/10.1037/met0000066

Besner, D., \& Coltheart, M. (1979). Ideographic and alphabetic processing in skilled reading of English. Neuropsychologia, 17(5), 467-472. https://doi.org/10.1016/00283932(79)90053-8

Campbell, J. I. D., \& Fugelsang, J. (2001). Strategy choice for arithmetic verification: effects of numerical surface form. Cognition, 80(3), B21-B30. https://doi.org/10.1016/s00100277(01)00115-9

Cohen Kadosh, R., Cohen Kadosh, K., Linden, D. E. J., Gevers, W., Berger, A., \& Henik, A. (2007). The brain locus of interaction between number and size: A combined functional magnetic resonance imaging and event-related potential study. Journal of Cognitive Neuroscience, 19(6), 957-970. https://doi.org/10.1162/jocn.2007.19.6.957

Farrell, S., \& Ludwig, C. J. H. (2008). Bayesian and maximum likelihood estimation of hierarchical response time models. Psychonomic Bulletin \& Review, 15(6), 1209-1217. https://doi.org/10.3758/pbr.15.6.1209

Faulkenberry, T. J., Cruise, A., Lavro, D., \& Shaki, S. (2016). Response trajectories capture the continuous dynamics of the size congruity effect. Acta Psychologica, 163, 114123. https://doi.org/10.1016/j.actpsy.2015.11.010 
Faulkenberry, T. J. (2017). A single-boundary accumulator model of response times in an addition verification task. Frontiers in Psychology, 8(1225), 1-12. https://doi.org/10.3389/fpsyg.2017.01225

Faulkenberry, T., Vick, A., \& Bowman, K. (2018). A shifted Wald Decomposition of the numerical size-congruity effect: Support for a late interaction account. Polish Psychological Bulletin 49(4), 391-397. https://doi.org/10.24425/119507

Faulkenberry, T. J., Ly, A., \& Wagenmakers, E. J. (2020). Bayesian inference in numerical cognition: A tutorial using JASP. Journal of Numerical Cognition, 6(2), 231-259. https://doi.org/10.5964/jnc.v6i2.288

Fitousi, D., \& Algom, D. (2006). Size congruity effects with two-digit numbers: Expanding the number line? Memory \& Cognition, 34(2), 445-457. https://doi.org/10.3758/bf03193421

Frampton, A. R., \& Faulkenberry, T. J. (2018). Mental arithmetic processes: Testing the independence of encoding and calculation. Journal of Psychological Inquiry, 22, 30-35. https://www.psychinquiry.org/wp-content/uploads/2019/03/Vol22-1.pdf

Heathcote, A. (2004). Fitting Wald and ex-Wald distributions to response time data: An example using functions for the S-PLUS package. Behavior Research Methods, Instruments, \& Computers, 36(4), 678-694. https://doi.org/10.3758/bf03206550

Henik, A., \& Tzelgov, J. (1982). Is three greater than five: The relation between physical and semantic size in comparison tasks. Memory \& Cognition, 10(4), 389-395. https://doi.org/10.3758/bf03202431

Hohle, R. H. (1965). Inferred components of reaction times as functions of foreperiod duration. Journal of Experimental Psychology, 69(4), 382-386. https://doi.org/10.1037/h0021740 JASP Team (2020). JASP (Version 0.14) [Computer software]. 
Kass, R. E., \& Raftery, A. E. (1995). Bayes factors. Journal of the American Statistical Association, 90(430), 773-795. https://doi.org/10.2307/2291091

Link, S. W. (1975). The relative judgment theory of two choice response time. Journal of Mathematical Psychology, 12(1), 114-135. https://doi.org/10.1016/0022-2496(75)90053$\mathrm{x}$

Luce, R. (1986). Response Times: Their Role in Inferring Elementary Mental Organization. New York, NY: Oxford University Press

MacLeod, C. M. (1991). Half a century of research on the Stroop effect: An integrative review. Psychological Bulletin, 109(2), 163-203. https://doi.org/10.1037/00332909.109.2.163

Masson, M. E. J. (2011). A tutorial on a practical Bayesian alternative to null-hypothesis significance testing. Behavior Research Methods, 43(3), 679-690. https://doi.org/10.3758/s13428-010-0049-5

Mathôt, S., Schreij, D., \& Theeuwes, J. (2012). OpenSesame: An open-source, graphical experiment builder for the social sciences. Behavior Research Methods, 44(2), 314324. https://doi.org/10.3758/s13428-011-0168-7

Matzke, D., \& Wagenmakers, E. J. (2009). Psychological interpretation of the ex-Gaussian and shifted Wald parameters: A diffusion model analysis. Psychonomic Bulletin \& Review, 16(5), 798-817. https://doi.org/10.3758/pbr.16.5.798

Myung, I. J. (2003). Tutorial on maximum likelihood estimation. Journal of Mathematical Psychology, 47(1), 90-100. https://doi.org/10.1016/s0022-2496(02)00028-7 
Penner-Wilger, M., Leth-Steensen, C., \& LeFevre, J. -A. (2002). Decomposing the problem-size effect: A comparison of response time distributions across cultures. Memory \& Cognition, 30(7), 1160-1167. https://doi.org/10.3758/bf03194333

Ratcliff, R. (1978). A theory of memory retrieval. Psychological Review, 85(2), 59-108. https://doi.org/10.1037/0033-295x.85.2.59

Ratcliff, R., \& Rouder, J. N. (2000). A diffusion model account of masking in two-choice letter identification. Journal of Experimental Psychology: Human Perception and Performance, 26(1), 127-140. https://doi.org/10.1037/0096-1523.26.1.127

Ratcliff, R., Thapar, A., \& McKoon, G. (2001). The effects of aging on reaction time in a signal detection task. Psychology and Aging, 16(2), 323-341. Doi:10.1037/0882-7974.16.2.323

Reike, D., \& Schwarz, W. (2017). Exploring the origin of the number-size congruency effect: Sensitivity or response bias? Attention, Perception, \& Psychophysics, 79(2), 383-388. https://doi.org/10.3758/s13414-016-1267-4

Rieger, T., \& Miller, J. (2019). Are model parameters linked to processing stages? An empirical investigation for the ex-Gaussian, ex-Wald, and EZ diffusion models. Psychological Research, 84(6), 1683-1699. https://doi.org/10.1007/s00426-019-01176-4

Risko, E. F., Maloney, E. A., \& Fugelsang, J. A. (2013). Paying attention to attention: evidence for an attentional contribution to the size congruity effect. Attention, Perception, \& Psychophysics, 75(6), 1137-1147. https://doi.org/10.3758/s13414-013-0477-2

Rouder, J. N. (2015). The what, why, and how of born-open data. Behavior Research Methods, 48(3), 1062-1069. https://doi.org/10.3758/s13428-015-0630-z 
Rouder, J. N., Speckman, P. L., Sun, D., Morey, R. D., \& Iverson, G. (2009). Bayesian $t$ tests for accepting and rejecting the null hypothesis. Psychonomic Bulletin \& Review, 16(2), 225237. https://doi.org/10.3758/pbr.16.2.225

Santens, S., \& Verguts, T. (2011). The size congruity effect: Is bigger always more? Cognition, 118(1), 94-110. https://doi.org/10.1016/j.cognition.2010.10.014

Schwarz, W., \& Heinze, H. (1998). On the interaction of numerical and size information in digit comparison: a behavioral and event-related potential study. Neuropsychologia, 36(11), 1167-1179. https://doi.org/10.1016/s0028-3932(98)00001-3

Schwarz, W. (2001). The ex-Wald distribution as a descriptive model of response times. Behavior Research Methods, Instruments, \& Computers, 33(4), 457-469. https://doi.org/10.3758/bf03195403

Sobel, K. V., Puri, A. M., \& Faulkenberry, T. J. (2016). Bottom-up and top-down attentional contributions to the size congruity effect. Attention, Perception, \& Psychophysics, 78(5), 1324-1336. https://doi.org/10.3758/s13414-016-1098-3

Sobel, K. V., Puri, A. M., Faulkenberry, T. J., \& Dague, T. D. (2017). Visual search for conjunctions of physical and numerical size shows that they are processed independently. Journal of Experimental Psychology: Human Perception and Performance, 43(3), 444-453. https://doi.org/10.1037/xhp0000323

Stroop, J. R. (1935). Studies of interference in serial verbal reactions. Journal of Experimental Psychology, 18(6), 643-662. https://doi.org/10.1037/h0054651

Wagenmakers, E.-J. (2007). A practical solution to the pervasive problems of $p$ values. Psychonomic Bulletin \& Review, 14(5), 779-804. https://doi.org/10.3758/bf03194105 
Wagenmakers, E.-J., Van Der Maas, H. L. J., \& Grasman, R. P. P. P. (2007). An EZ-diffusion model for response time and accuracy. Psychonomic Bulletin \& Review, 14(1), 3-22. https://doi.org/10.3758/bf03194023

Wagenmakers, E.-J., Ratcliff, R., Gomez, P., \& McKoon, G. (2008). A diffusion model account of criterion shifts in the lexical decision task. Journal of Memory and Language, 58(1), 140-159. https://doi.org/10.1016/j.jml.2007.04.006

Walsh, V. (2003). A theory of magnitude: common cortical metrics of time, space and quantity. Trends in Cognitive Sciences, 7(11), 483-488. https://doi.org/10.1016/j.tics.2003.09.002

Whelan, R. (2008). Effective analysis of reaction time data. The Psychological Record, 58(3), 475-482. https://doi.org/10.1007/bf03395630

Voss, A., Rothermund, K., \& Voss, J. (2004). Interpreting the parameters of the diffusion model: An empirical validation. Memory \& Cognition, 32(7), 1206-1220. https://doi.org/10.3758/bf03196893 
Table 1

Descriptive statistics for each response time model

\begin{tabular}{|c|c|c|c|c|c|c|}
\hline \multirow[t]{2}{*}{ ex-Gaussian } & \multicolumn{2}{|c|}{$\mu$} & \multicolumn{2}{|c|}{$\sigma$} & \multicolumn{2}{|c|}{$\tau$} \\
\hline & $M$ & $S D$ & $M$ & $S D$ & $M$ & $S D$ \\
\hline congruent & 425.3 & 64.03 & 46.24 & 15.69 & 128.9 & 52.25 \\
\hline incongruent & 443.6 & 75.21 & 57.09 & 22.56 & 176.6 & 58.10 \\
\hline
\end{tabular}

\begin{tabular}{lcccccccc} 
ex-Wald & \multicolumn{2}{c}{ Drift rate } & & \multicolumn{2}{c}{ Response threshold } & & \multicolumn{2}{c}{ Nondecision time } \\
& $M$ & $S D$ & & $M$ & $S D$ & & $M$ & $S D$ \\
\cline { 2 - 3 } congruent & 0.4495 & 0.1402 & & 187.9 & 49.31 & & 123.4 & 42.29 \\
incongruent & 0.3808 & 0.1567 & & 164.5 & 52.17 & & 163.8 & 46.02
\end{tabular}

\begin{tabular}{lcccccccc} 
shifted Wald & \multicolumn{2}{c}{ Drift rate } & & \multicolumn{2}{c}{ Response threshold } & & \multicolumn{2}{c}{ Nondecision time } \\
& $M$ & $S D$ & & $M$ & $S D$ & & $M$ & $S D$ \\
\cline { 2 - 3 } congruent & 0.1341 & 0.03056 & & 39.68 & 14.99 & & 252.1 & 63.14 \\
incongruent & 0.1094 & 0.02043 & & 41.28 & 15.03 & & 238.0 & 57.11
\end{tabular}

\begin{tabular}{lcccccccc} 
EZ-diffusion & \multicolumn{2}{c}{ Drift rate } & & \multicolumn{2}{c}{ Response threshold } & & \multicolumn{2}{c}{ Nondecision time } \\
& $M$ & $S D$ & & $M$ & $S D$ & & $M$ & $S D$ \\
\cline { 2 - 4 } congruent & 0.01102 & 0.001515 & & 5.489 & 0.8132 & & 296.2 & 55.47 \\
incongruent & 0.00951 & 0.001163 & & 6.283 & 0.7705 & & 282.0 & 68.73 \\
\hline
\end{tabular}

Note. $M=$ mean, $S D=$ standard deviation 


\section{Figure 1.}

The shifted Wald as a cognitive model, describing RT as the time for an accumulator to drift toward and hit a single boundary $\alpha$ at rate $\gamma=2.0$. The nondecision time $\theta=0.15$ represents the component of RT which is not due to this accumulation process. The solid black line represents the accumulator for a single trial, whereas the dashed upper curve represents the shifted Wald distribution formed by collecting RTs for many such trials.

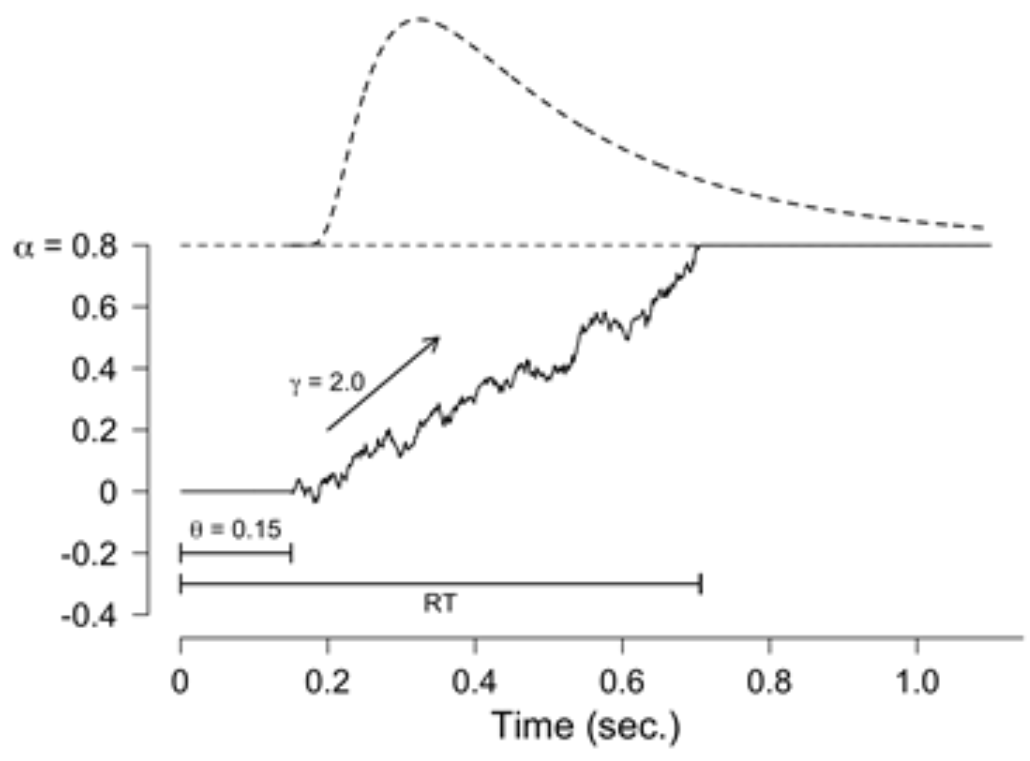




\section{Figure 2.}

Example stimuli in a physical size comparison task. The left panel depicts a congruent trial, where the physically larger number (8) is also the numerically larger digit. The right panel depicts an incongruent trial, where the physically larger number (2) is the numerically smaller digit.

Congruent

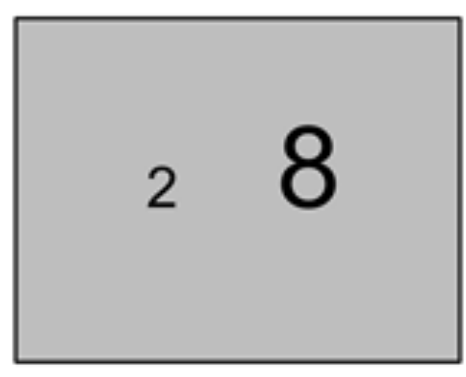

Incongruent

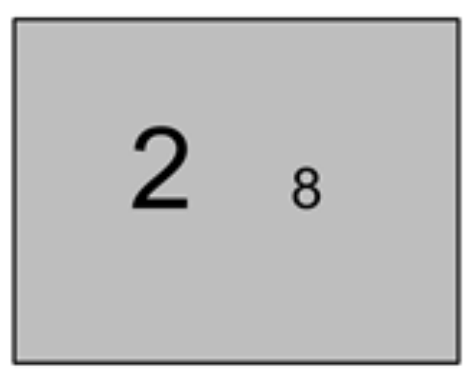


Figure 3.

Sequence of experimental trials.
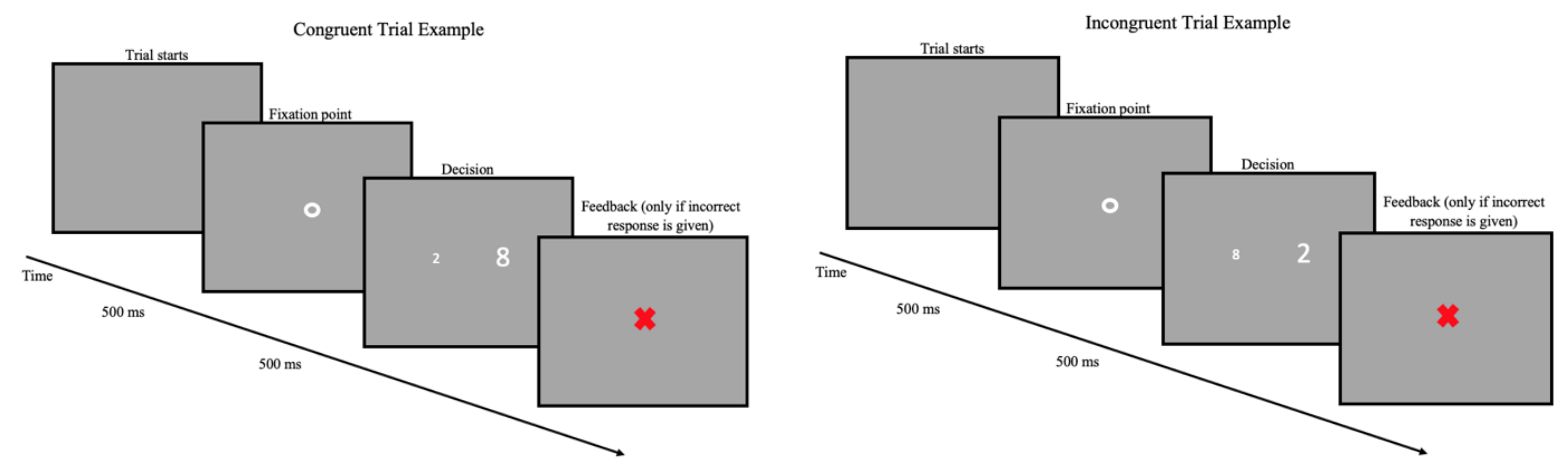
Figure 4 .

Distributions of response times (in seconds) as a function of congruity (congruent versus incongruent).

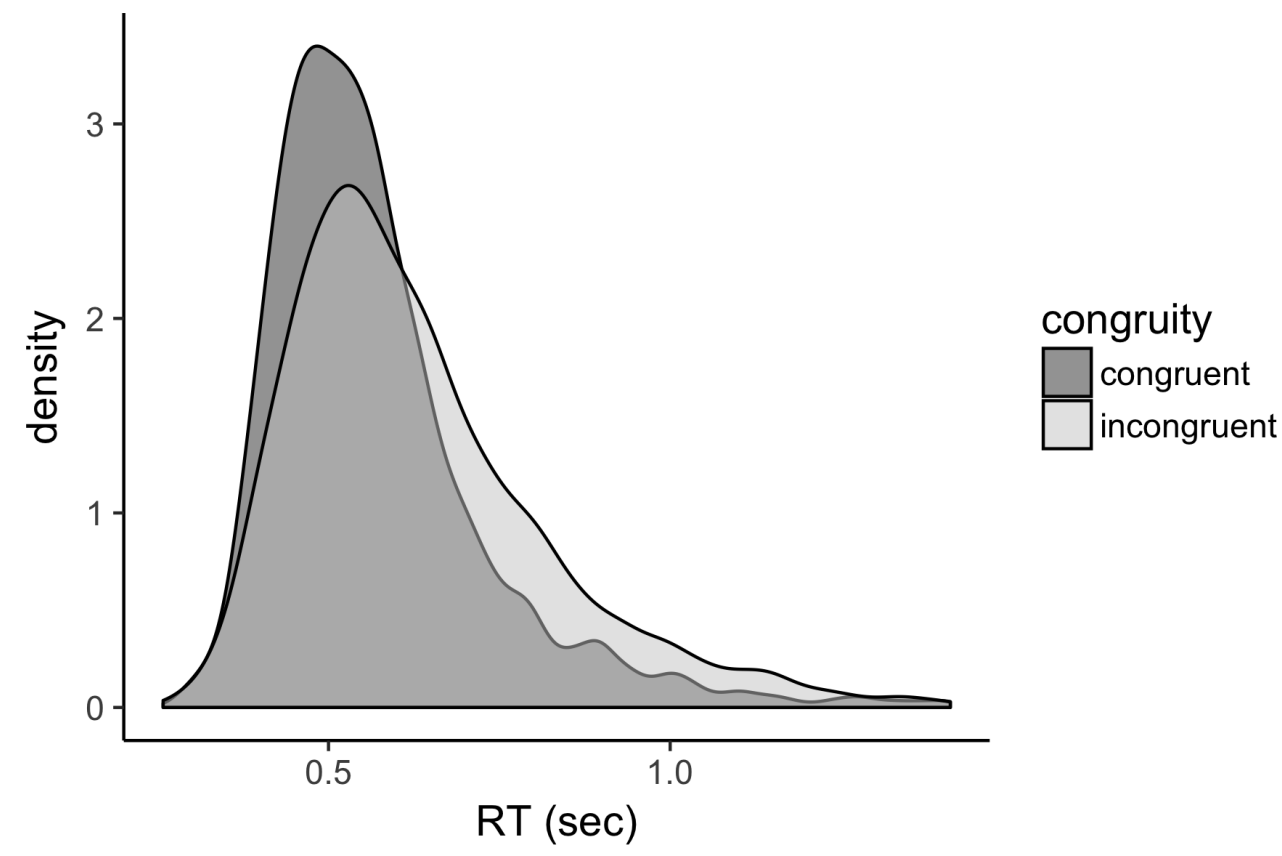

